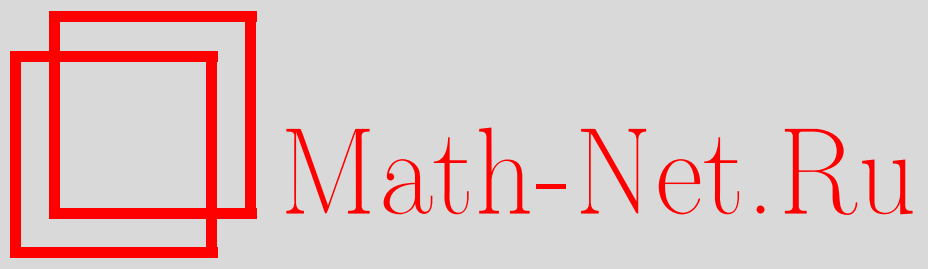

А. В. Дудко, Н. И. Нессонов, Проективные характеры бесконечной обобщенной симметрической группы, Функи. анализ и его прил., 2008, том 42, выпуск 1, 82-85

DOI: https://doi.org/10.4213/faa2893

Использование Общероссийского математического портала MathNet.Ru подразумевает, что вы прочитали и согласны с пользовательским соглашением

http: //www. mathnet.ru/rus/agreement

Параметры загрузки:

IP : 54.237 .59 .107

26 апреля 2023 г., 08:15:59

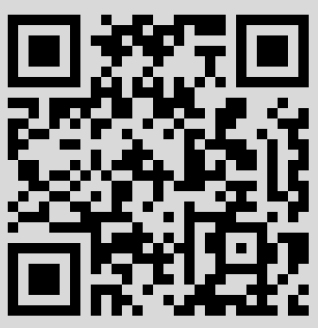


представления, Наука, М., 1974. [4] Ю. Д. Латушкин, А. М. Степин, УМН, 46:2 (1991), 85-143. [5] 3. Нитецки, Введение в дифференциальную динамику, Мир, М., 1975. [6] J. M. G. Fell, Acta Math., 106:3-4 (1961), 233-280. [7] M. J. Dupré, R. M. Gillette, Banach Bundles, Banach Modules and Automorphisms of $C^{*}$-Algebras, Pitman Publishing Inc., Boston, 1983. [8] A. Grothendieck, in: Dix Exposes sur la cohomology des schemas, North-Holland, Amsterdam; Masson, Paris, 1968, 46-66.

Белорусский государственный университет

Университет в Белостоке

Поступило в редакцию e-mail: antonevich@bsu.by 25 августа 2006 г.

Белорусский государственный университет e-mail: itrubnikov@gmail.com

УДК 512.547.4

\title{
Проективные характеры бесконечной обобщенной симметрической группы
}

\author{
(c) 2008. А. В. Дудко, Н. И. Нессонов
}

Обобщенная симметрическая группа $B_{m}^{\infty}$ по определению является полупрямым произведением $S(\infty) \ltimes \mathbb{Z}_{m}^{\infty}$, где $S(\infty)$ - группа всех конечных перестановок натурального ряда, а $\mathbb{Z}_{m}^{\infty}$ - множество последовательностей элементов из $\mathbb{Z}_{m}$ с конечным числом ненулевых координат, на котором задано естественное действие группы $S(\infty)$. В данной заметке анонсируется полная классификация проективных факторпредставлений конечного типа группы $B_{m}^{\infty}$, которым с помощью известной конструкции (см. [4]) сопоставляются обычные факторпредставления ее накрывающей

$$
\begin{gathered}
\widetilde{B}_{m}^{\infty}=\left\langle\tilde{s}_{i}, \widetilde{w}_{j}(i, j \in \mathbb{N})\right| \tilde{s}_{i}^{2}=1 ; \widetilde{w}_{i}^{m}=1 ;\left(\tilde{s}_{i} \tilde{s}_{i+1}\right)^{3}=1 ; \tilde{s}_{i} \widetilde{w}_{i}=\widetilde{w}_{i+1} \tilde{s}_{i} ; \\
\left.\tilde{s}_{i} \widetilde{w}_{j}=\vartheta \widetilde{w}_{j} \tilde{s}_{i}, j \neq i, i+1 ;\left(\tilde{s}_{i} \tilde{s}_{j}\right)^{2}=\nu,|i-j|>1 ; \widetilde{w}_{i} \widetilde{w}_{j}=\mu \widetilde{w}_{j} \widetilde{w}_{i}, i \neq j\right\rangle .
\end{gathered}
$$

Здесь $\vartheta, \nu, \mu$ - центральные элементы, удовлетворяющие условию $\vartheta^{(2, m)}=$ $\nu^{2}=\mu^{(2, m)}=1$, где $(2, m)$ - наименьший общий делитель чисел 2 и $m$. Аналогичная задача для группы $S(\infty)$ решена Назаровым [5] (см. также [3]). Понятно, что для любого унитарного факторпредставления $\pi$ группы $\widetilde{B}_{m}^{\infty}$ операторы $\pi(\vartheta), \pi(\nu), \pi(\mu)$ могут отличаться только знаком от единичного оператора. С другой стороны, упорядоченный триплет $c(\pi)=(\pi(\vartheta), \pi(\nu), \pi(\mu))$ можно рассматривать как элемент второй группы когомологий

$$
\mathscr{H}^{2}\left(B_{m}^{\infty}, T\right)= \begin{cases}\mathbb{Z}_{2}^{3}, & \text { если } m=2 k(k \in \mathbb{N}), \\ \mathbb{Z}_{2}, & \text { если } m=2 k-1,\end{cases}
$$

где $T$ - одномерный тор, определяющий конкретное централъное расширение. При взятии тензорного произведения представлений соответствующие триплеты перемножаются.

1. Обычные представления группы $\boldsymbol{B}_{\boldsymbol{m}}^{\infty}$ описаны независимо в [1] и [2]. Для вычисления характера обычного представления параметризуем, в первую очередь, классы сопряженных элементов. Произвольный элемент $g \in B_{m}^{\infty}$ имеет 
вид $g=s w$, где $s \in S(\infty), w=\left(w_{i}^{d_{i}}\right) \in \mathbb{Z}_{m}^{\infty}$. Пусть $\mathbb{N} / s-$ множество орбит перестановки $s$ и $s^{(p)}$ - цикл, совпадающий с $s$ на орбите $p$. Для $p \in \mathbb{N} / s$ положим $w(p)=\prod_{i \in p} w_{i}^{d_{i}}$. Если $|p|$ - количество точек орбиты $p$, то неупорядоченное множество пар $\{(|p|, w(p))\}_{p \in \mathbb{N} / s}$ является полным инвариантом сопряженности в группе $B_{m}^{\infty}$.

Теорема 1. Пусть $\pi$ - бакторпредставление конечного типа группь $B_{m}^{\infty}$. Тогда существуют две последовательности $\alpha=\left\{\alpha_{i}\right\}, \beta=\left\{\beta_{i}\right\}$ невозрастающих положительных чисел, удовлетворяюших условию $\sum\left(\alpha_{j}+\beta_{j}\right) \leqslant 1$, две последовательности $\widehat{\alpha}=\left\{\widehat{\alpha}_{i}\right\}, \hat{\beta}=\left\{\hat{\beta}_{i}\right\}$ характеров группы $\mathbb{Z}_{m}$ и нормированная положительно определенная функиия $\zeta$ на $\mathbb{Z}_{m}$, такие, что значения соответствующего представлению $\pi$ характера $\chi_{\beta \hat{\beta}}^{\alpha \widehat{\alpha}}$ задаютсл формулой

$$
\chi_{\beta \hat{\beta}}^{\alpha \widehat{\alpha}}(s w)=\prod_{p \in \mathbb{N} / s}\left\{\sum_{j}\left[\alpha_{j}^{|p|} \widehat{\alpha}_{i}(w(p))+(-1)^{|p|-1} \beta_{j}^{|p|} \hat{\beta}_{i}(w(p))\right]+\delta_{p} \zeta(w(p))\right\},
$$

$2 \partial e$

$$
\delta_{p}= \begin{cases}1-\sum\left(\alpha_{j}+\beta_{j}\right) & \text { nрu }|p|=1 \\ 0 & \text { nрu }|p|>1\end{cases}
$$

2. Базисные представления группы $\widetilde{\boldsymbol{B}}_{\boldsymbol{m}}^{\infty}$. Пусть $\mathscr{E}=\left[\begin{array}{ll}1 & 0 \\ 0 & 1\end{array}\right], \mathscr{I}=$ $\left[\begin{array}{ll}0 & 1 \\ 1 & 0\end{array}\right], \mathscr{J}=\left[\begin{array}{ll}0 & i \\ i & 0\end{array}\right], \mathscr{K}=\left[\begin{array}{cc}1 & 0 \\ 0 & -1\end{array}\right]$ - элементы алгебры $2 \times 2$-комплексных матриц $M_{2}(\mathbb{C})$ и $\operatorname{tr}_{2}$ - нормированный след на $M_{2}(\mathbb{C})$. Зададим представление $\digamma$ на образующих, положив $\digamma\left(\tilde{s}_{k}\right)=\mathscr{I}, \digamma\left(\widetilde{w}_{k}\right)=(-1)^{k} \mathscr{J}$. Так как $\mathscr{J} \mathscr{I}=$ $-\mathscr{I} \mathscr{J}$, то $c(\digamma)=(I,-I, I)$. Пусть, далее, $B=\bigotimes_{i \in \mathbb{N}} M_{i}$ и $\tau=\bigotimes_{i \in \mathbb{N}} \tau_{i}$, где $M_{i}=$ $M_{2}(\mathbb{C}), \tau_{i}=\operatorname{tr}_{2}, \mathscr{M}_{2 n-1}=\mathscr{K}^{\otimes(n-1)} \otimes \mathscr{I} \otimes \mathscr{E}^{\otimes \infty}, \mathscr{M}_{2 n}=\mathscr{K}^{\otimes(n-1)} \otimes \mathscr{J} \otimes \mathscr{E}^{\otimes \infty}$. Мы рассматриваем $B$ как $\star$-подалгебру $\mathrm{II}_{1}$-фактора $M$, построенного по представлению Гельфанда-Наймарка-Сигала алгебры $B$ для состояния $\tau$. Определим отображения $\Pi, \Phi: \widetilde{B}_{m}^{\infty} \rightarrow B$ на образующих с помощью следующих соотношений:

$$
\begin{gathered}
\Pi\left(\tilde{s}_{k}\right)=\frac{1}{\sqrt{2}}\left(\mathscr{M}_{k}-\mathscr{M}_{k+1}\right), \quad \Pi\left(\widetilde{w}_{k}\right)=(-1)^{k} \mathscr{M}_{k}, \\
\Phi\left(\tilde{s}_{k}\right)=\frac{1}{\sqrt{2 k}}\left(\sqrt{k-1} \mathscr{M}_{k-1}-\sqrt{k+1} \mathscr{M}_{k}\right) \quad\left(\text { cм. [5]), } \Phi\left(\widetilde{w}_{k}\right)=\mathscr{E} \otimes \infty .\right.
\end{gathered}
$$

Прямая проверка показывает, что П и $\Phi$ расширяются до $\mathrm{II}_{1}$-факторпредставлений группы $\widetilde{B}_{m}^{\infty}$, для которых $c(\Pi)=(-I,-I,-I), c(\Phi)=(-I, I, I)$. В случае нечетного $m$ ввиду условий на центральные элементы $\vartheta, \nu, \mu$ из формулы $(1)$ остается только одно представление $\Phi$. Любой другой неединичный триплет $c(T)$ можно получить в виде произведения не более трех разных множителей $c(\Pi), c(\Phi), c(\digamma)$. Соответствующие характеры тензорных произведений представлений П, $\Phi, \digamma$ и представления Гельфанда-Наймарка-Сигала, построенные по ним, будем называть базисными.

Теорема 2. Базисные представления являются факторпредставлениями.

3. Классификация характеров группы $\widetilde{\boldsymbol{B}}_{\boldsymbol{m}}^{\infty}$. Рассматривая $B_{m}^{\infty}$ как группу с образующими $s_{i}, w_{j}$, удовлетворяющими соотношениям для $\tilde{s}_{i}, \widetilde{w}_{j}$ из $(1)$ при $\vartheta=\nu=\mu=1$, определим гомоморфизм $\mathfrak{p r}: \widetilde{B}_{m}^{\infty} \rightarrow B_{m}^{\infty}$ на образующих: 
$\mathfrak{p r}(v)=1$ для $v=\vartheta, \nu, \mu, \mathfrak{p r}\left(\tilde{s}_{i}\right)=s_{i}$ и $\mathfrak{p r}\left(\widetilde{w}_{i}\right)=w_{i}$. Если $\mathfrak{b} \in \widetilde{B}_{m}^{\infty}, \mathfrak{p r}(\mathfrak{b})=s w$, то $\mathfrak{b}=\prod_{p \in \mathbb{N} / s} \mathfrak{b}_{p}$, где $\mathfrak{b}_{p} \in \mathfrak{p r}^{-1}\left(s^{(p)} w(p)\right)$.

Теорема 3. Пусть $\pi$ - бакторпредставление конечного типа группь $\widetilde{B}_{m}^{\infty}$, $\chi_{\pi}$ - соответствующий след $u \pi_{b}$ - базисное представление, для которого $c\left(\pi_{b}\right)=c(\pi)$. Тогда существуют $\alpha, \beta, \widehat{\alpha}, \hat{\beta}$ (см. теорему 1$)$, такие, что

$$
\chi_{\pi}(\mathfrak{b})=\chi_{\pi_{b}}(\mathfrak{b}) \chi_{\beta \hat{\beta}}^{\alpha \widehat{\alpha}}(\mathfrak{p r}(\mathfrak{b})), \quad \text { əде } \mathfrak{b} \in \widetilde{B}_{m}^{\infty} .
$$

Заметим, что при заданных $\chi_{\pi}$ и $\chi_{\pi_{b}}$ характер $\chi_{\beta \hat{\beta}}^{\alpha \widehat{\alpha}}$ из последнего соотношения определяется неединственным образом. Эта неоднозначность связана со свойствами множества нулей функции $\chi_{\pi}$ и для каждого из расширений описывается явно.

Набросок доказательства теоремы 3. Характеры факторпредставлений называют неразложимыми. Доказательство неразложимости характера $\chi_{\pi}$, определенного согласно (5), опирается на теорему 2 и использует соображения мультипликативности.

Основную идею доказательства обратного утверждения мы, опуская технические построения, объясним для триплетов $(-I, I, I)$ и $(-I,-I, I)$.

Если $\pi$ - факторпредставление группы $\widetilde{B}_{m}^{\infty}$ и $c(\pi)=(-I, I, I)$, то, опираясь на свойства классов сопряженных элементов, можно показать, что

$\chi_{\pi}(\mathfrak{b})=0$, когда перестановка $s$ из $s w=\mathfrak{p r}(\mathfrak{b})$ имеет цикл четной длины.

Отсюда можно вывести свойство мулътипликативности характера $\chi_{\pi}: \chi_{\pi}(\mathfrak{b})=$ $\prod_{p \in \mathbb{N} / s} \chi_{\pi}\left(\mathfrak{b}_{p}\right)$, которое в случае $(-I,-I, I)$ нарушается. Следовательно, представление Гельфанда-Наймарка-Сигала, построенное по $\chi_{\Phi} \otimes \chi_{\pi}$, является обычным факторпредставлением группы $B_{m}^{\infty}$. Согласно теореме $1, \chi_{\Phi} \otimes \chi_{\pi}=\chi_{\beta \hat{\beta}}^{\alpha \widehat{\alpha}}$. Из (6) можно получить следующие важные соотношения:

$$
\left\{\mathfrak{b} \in \widetilde{B}_{m}^{\infty} \mid \chi_{\Phi}(\mathfrak{b})=0\right\} \subset\left\{\mathfrak{b} \in \widetilde{B}_{m}^{\infty} \mid \chi_{\pi}(\mathfrak{b})=0\right\}, \quad \alpha_{k}=\beta_{k}, \widehat{\alpha}_{k}=\hat{\beta}_{k} \forall k .
$$

Таким образом, $\chi_{\pi}$ однозначно восстанавливается по $\chi_{\beta \hat{\beta}}^{\alpha \widehat{\alpha}}$. Опираясь на $(7)$, с помощью несложных вычислений можно убедиться, что $\chi_{\pi}=\chi_{\Phi} \otimes \chi_{0}^{2 \alpha \widehat{\alpha}}$.

При доказательстве аналогов свойств (6), (7) для факторпредставления $\pi$ с $c(\pi)=(-I,-I, I)$ мы, следуя подходу Окунькова [6], вводим операторы $\mathscr{O}_{i}=$ $\mathrm{s} \lim _{n \rightarrow \infty} n^{-1} \sum_{k=i+1}^{i+n} t_{(i k)}$, где $t_{(i k)}=(-1)^{k-i-1} \pi\left(\tilde{s}_{k-1} \cdots \tilde{s}_{i+1} \tilde{s}_{i} \tilde{s}_{i+1} \cdots \tilde{s}_{k-1}\right)$. Если $\xi$ - циклический отделяющий вектор для $\pi$, соответствующий следу $\chi_{\pi}$, и $\mathfrak{p r}(\mathfrak{b})=s w$, то для любого $i(p)$ из орбиты $p$ перестановки $s$

$$
\chi_{\pi}(\mathfrak{b})=(\pi(\mathfrak{b}) \xi, \xi)= \pm\left(\mathscr{O}_{i\left(p_{1}\right)}^{\left|p_{1}\right|-1} \pi\left(\widetilde{w}_{i\left(p_{1}\right)}\right)^{d\left(p_{1}\right)} \mathscr{O}_{i\left(p_{2}\right)}^{\left|p_{2}\right|-1} \pi\left(\widetilde{w}_{i\left(p_{2}\right)}\right)^{d\left(p_{2}\right)} \cdots \xi, \xi\right),
$$

где $\widetilde{w}=\prod_{i} \widetilde{w}_{i}^{d_{i}}, d(p)=\sum_{i \in p} d_{i}$. Далее доказываются следующие соотношения: $\pi\left(\tilde{s}_{k}\right) \mathscr{O}_{k+1}=-\mathscr{O}_{k} \pi\left(\tilde{s}_{k}\right) ; \pi\left(\tilde{s}_{k}\right) \mathscr{O}_{i}=-\mathscr{O}_{i} \pi\left(\tilde{s}_{k}\right), k \neq i-1, i ; \pi\left(\widetilde{w}_{k}\right) \mathscr{O}_{i}=-\mathscr{O}_{i} \pi\left(\widetilde{w}_{k}\right)$ для любых $i, k ; \mathscr{O}_{k} \mathscr{O}_{i}=-\mathscr{O}_{i} \mathscr{O}_{k}, i \neq k$. Отсюда и из (8) можно получить утверждение $(6)$ и включение из (7) ( $\Phi$ заменяется на $\Phi \otimes \digamma$ ). Теперь замечаем, что характеру $\chi_{1}=\chi_{\digamma} \otimes \chi_{\pi}$ соответствует представление $\pi_{1}$ с $c\left(\pi_{1}\right)=(-I, I, I)$, которое может и не быть фактором. Но фактором является $W^{*}$-алгебра, порожденная $\digamma\left(\widetilde{B}_{m}^{\infty}\right) \otimes I$ и $I \otimes \pi\left(\widetilde{B}_{m}^{\infty}\right)$. Отсюда, опираясь на вид представления 
$\digamma$, можно вывести, что $\chi_{1}$ есть среднее не более чем двух неразложимых характеров $\chi=\chi_{\Phi} \otimes \chi_{0}^{\alpha \widehat{\alpha}}$ и $\chi^{\prime}=\chi_{\Phi} \otimes \chi_{0}^{\alpha^{\prime} \widehat{\alpha}^{\prime}}$, где $\alpha_{k}=\alpha_{k}^{\prime}$, а $\widehat{\alpha}_{k}\left(w_{i}\right)=-\widehat{\alpha}_{k}^{\prime}\left(w_{i}\right)$ на образующей $w_{i} \in \mathbb{Z}_{m}$. Теперь утверждение теоремы для рассматриваемого случая вытекает из соотношения

$$
\chi_{1}=\chi_{\digamma} \otimes \chi_{\pi}=\chi_{\digamma} \otimes \chi_{\Phi} \otimes \chi_{0}^{\alpha \widehat{\alpha}}=\chi_{\digamma} \otimes \chi_{\Phi} \otimes \chi_{0}^{\alpha^{\prime} \widehat{\alpha}^{\prime}}
$$

Мы благодарны рецензенту за ряд важных рекомендаций и замечаний.

\title{
ЛитерАТУРА
}

[1] R. Boyer, Int. J. Math. Math. Sci., 9 (2005), 1365-1379. [2] A. Dudko, N. Nessonov, http://arxiv.org/abs/math/0510597. [3] В. Н. Иванов, Зап. научн. сем. ПОМИ, 240 (1997), 115-135; http://arxiv.org/abs/math/0303169. [4] A. Morris, H. Jones, Sem. Lothar. Combin., 50, Art. B50b (2003). [5] M. L. Nazarov, in: Representation Theory and Dynamical Systems, Adv. Soviet Math., vol. 9, Amer. Math. Soc., Providence, R.I., 1992, 115-130. [6] А. Ю. Окуньков, Функц. анализ и его прил., 28:2 (1994), 31-40.

Физико-технический институт низких температур, Харьков Поступило в редакцию e-mail: artemdudko@rambler.ru

12 мая 2006 г.

Физико-технический институт низких температур, Харьков e-mail: nessonov@ilt.kharkov.ua

УДК 517.986.4

\section{Операторы Рака для группы движений*}

\author{
(c) 2008. Р. С. ИСМАГИЛОВ
}

Введение. Классическим коэффициентам Рака посвящено большое число работ, многие из которых связаны с физическими приложениями (см. [1]-[4]). В работе автора [5] в качестве обобщения коэффициентов Рака дано определение операторов Рака для унитарных неприводимых представлений локальнокомпактных групп типа I. В данной работе мы воспроизведем это определение для представлений группы $G$, состоящей из движений евклидова пространства $\mathbb{R}^{3}$. Затем мы укажем явный вид операторов Рака для этой группы.

1. Представления группы $G$ и их тензорные произведения. Начнем с обозначений. Элементы группы $G$ записываем в виде $g=(u, a)$, где $u \in S O(3), a \in \mathbb{R}^{3}$ (таким образом, $g$ - это движение $x \mapsto x u+a$ ). Подгруппа $S O(3)$ действует на сфере $S^{2}=\left\{x \in \mathbb{R}^{3}:(x, x)=1\right\}$ по формуле $x \mapsto x u$; стабилизатор любой точки изоморфен группе $S O(2)$ (отождествим эту группу с $\left.T^{1}=\{z \in \mathbb{C}:|z|=1\}\right)$. Любое неприводимое унитарное представление группы $G$, нетривиальное на подгруппе $\mathbb{R}^{3} \subset G$, задается парой $(m, l)$, где $m \in \mathbb{Z}$ и $l>0$; обозначим его через $T^{m, l}$. Оно реализуется в пространстве $L_{2}\left(S^{2}\right)$; на подгруппе $\mathbb{R}^{3}$ это представление сводится к умножению на функцию $x \mapsto \exp (i l(a, x))$, а на подгруппе $S O(3)$ оно индуцировано характером $z \mapsto z^{m}$ группы $T^{1}$. 